\title{
SOME ASPECTS OF VUV BEAM LINE DESIGN
}

\author{
A. Gaupp and W. Peatman \\ Berliner Elektronenspeicherring-Gesellschaft für Synchrotronstrahlung $\mathrm{mbH}$ \\ (BESSY), Lentzeallee 100, 14195 Berlin, Germany
}

Some aspects of the design and usage of vacuum ultra violet beam lines are discussed. Fermat's principle for imaging is introduced and applied to grating monochromators. Some typical vacuum ultraviolet beam lines are presented, and some further topics believed to be of importance today and in the future are mentioned.

PACS numbers: 41.60.Ap, 42.15.-i

\section{Introduction}

The spectral range of the vacuum ultra violet (VUV) and soft X-ray region (XUV) is taken to be between 5 and $3000 \mathrm{eV}$ photon energy. In this range air is strongly absorbing, transmitting materials are not available, optical elements are usually of the reflective type, and wavelength selection is done by gratings. For acceptable reflection coefficients grazing incidence must be used above $35 \mathrm{eV}$ introducing optical aberrations.

The synchrotron radiation (SR) source is characterised being broad band, of high intensity, emitting from a small area into a small solid angle; the latter three properties make SR to have a high phase space density or high brilliance.

A SR beam line must transport the radiation from the source to the experiment. It must provide for wavelength selection and focussing. The design of such beam lines is the topic of this contribution. However, we only can touch upon some aspects. It takes a book to cover the field [1].

\section{Reflection}

In the VUV most materials are strongly absorbing. In order to manipulate the radiation mirrors are used. Reflecting surfaces are made from $\mathrm{Au}, \mathrm{Ni}, \mathrm{Pt}, \mathrm{Os}$, $\mathrm{W}, \mathrm{Al}$, or $\mathrm{SiC}$, to name but a few. The reflectivity of an Au surface versus photon energy for different incident angles is shown in Fig. 1 [2].

At a real mirror the reflectivity is reduced due to scattering by a rough surface, by matter contaminating the optical surface etc. The necessity to use grazing incidence reduces the acceptance of a given mirror and introduces aberrations while at the same time it reduces the effect of surface roughness. 


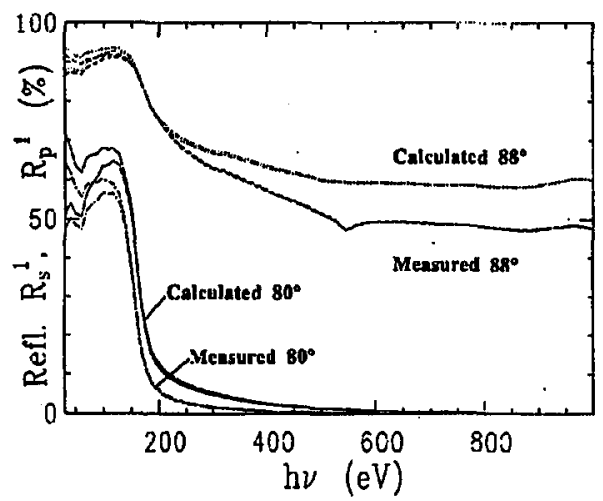

Fig. 1. Calculated and measured reflectivity for $s$ - and $p$-reflection by a gold surface.

\section{Fermat's principle}

We base the description of the light path on Fermat's principle [3]. It does not include diffraction effects. Among all possible paths light follows that one that is stationary against variations of the path. We restrict ourselves to propagation in a medium with a constant index of refraction taken to be $n=1$, where light travels along straight lines.

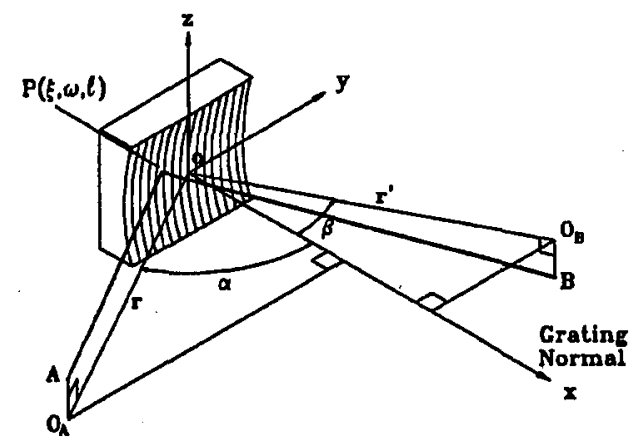

Fig. 2. Reflection by a mirror.

The situation is shown in Fig. 2. According to Fermat's principle only those paths occur that are of equal length. From wave optics it is evident that "equal" means "equal within a quarter wavelength". If all points $P$ on the mirror connect $A$ and $B$ with the same path length all light coming from $A$ is focussed at $B$, and the image is perfect. A path linking $A$ and $B$ is uniquely described by the coordinates of $P$. The path length is

$$
\begin{aligned}
F= & \overline{A P}+\overline{P B}=\sqrt{(x-\xi)^{2}+(y-\omega)^{2}+(z-l)^{2}} \\
& +\sqrt{\left(x^{\prime}-\xi\right)^{2}+\left(y^{\prime}-\omega\right)^{2}+\left(z^{\prime}-l\right)^{2}} .
\end{aligned}
$$


This path is an extremum for

$$
\frac{\partial F}{\partial \omega}=0, \quad \frac{\partial F}{\partial l}=0 .
$$

We expand $F$ into a Taylor series with respect to $\omega$ and $l$ with coefficients $F_{i j k}$ which should rapidly converge for the small opening angles employed in the VUV [4]. Coefficients with $k \neq 0$ depend on $z$ and $z^{\prime}$.

The optical surface is described by a second Taylor expansion [5] (see Fig. 2)

$$
\xi=\sum_{i=0}^{\infty} \sum_{j=0}^{\infty} a_{i j} w^{i} l^{j}
$$

The expansion coefficients of the optical path are listed in Table, where the following substitution has been made introducing the angles with respect to the mirror normal:

$$
x=r \cos \alpha, \quad y=r \sin \alpha, \quad x^{\prime}=r^{\prime} \cos \beta, \quad y^{\prime}=r^{\prime} \sin \beta .
$$

A perfect image results if all coefficients $F_{i j k}$ vanish except $F_{000}$. In general the dominating terms are $F_{200}$ and $F_{020}$. These coefficients vanish at the meridional and sagittal focus, respectively.

TABLE

Values of $F_{i j k}$.

\begin{tabular}{l|l}
\hline \hline$F_{000}=r+r^{\prime}$, & \\
$F_{100}=N k \lambda-(\sin \alpha+\sin \beta)$, & grating term \\
$F_{200}=\frac{\cos ^{2} \alpha}{r}+\frac{\cos ^{2} \beta}{r^{\prime}}-2 a_{20}(\cos \alpha+\cos \beta)$, & meridional focal term \\
$F_{020}=\frac{1}{r}+\frac{1}{r^{\prime}}-2 a_{02}(\cos \alpha+\cos \beta)$, & sagittal focal term \\
$F_{300}=\frac{T(r, \alpha)}{r} \sin \alpha+\frac{T\left(r^{\prime}, \beta\right)}{r^{\prime}} \sin \beta-2 a_{30}(\cos \alpha+\cos \beta)$, & primary coma term \\
$F_{120}=\frac{S(r, \alpha)}{r} \sin \alpha+\frac{S\left(r^{\prime}, \beta\right)}{r^{\prime}} \sin \beta-2 a_{12}(\cos \alpha+\cos \beta)$, & astigmatic coma term \\
$F_{011}=-\frac{z}{r}-\frac{z^{\prime}}{r}$, & \\
$F_{111}=-\frac{z \sin \alpha}{r^{2}}-\frac{z^{\prime} \sin \beta}{r^{\prime 2}}$, & \\
$F_{102}=\frac{z^{2} \sin \alpha}{r^{2}}+\frac{z^{\prime 2} \sin \beta}{r^{\prime 2}}$, &
\end{tabular}

where $T(r, \alpha)=\frac{\cos ^{2} \alpha}{r}-2 a_{20} \cos \alpha$ and $S(r, \alpha)=\frac{1}{r}-2 a_{02} \cos \alpha$ with analogous expression for $T\left(r^{\prime}, \beta\right)$ and $S\left(r^{\prime}, \beta\right)$.

\section{Gratings}

The diffraction effects of the grating grooves is added to Fermat's principle by adding the phase advance per groove to the optical path

$$
F \rightarrow F+N k \lambda \omega,
$$

where $N$ is the grating line density, $\lambda$ is the wavelength, and $k$ is the order of spectrum. This adds to the term $F_{100}$. Then the coefficient of $\omega$ vanishes for

$$
0=N k \lambda-(\sin \alpha+\sin \beta),
$$

which is known as the grating equation. Defocussing effects at a grating cause a spectral line width of 


$$
\begin{aligned}
\Delta \lambda= & \frac{1}{N k}\left[\omega F_{200}+\frac{3}{2} \omega^{2} F_{300}+\frac{1}{2} l^{2} F_{120}+\frac{1}{2} \omega^{3} F_{400}\right. \\
& \left.+\frac{1}{2} \omega l^{2} F_{220}+l F_{111}+\frac{1}{2} F_{102}+\frac{1}{2} \omega F_{202}+\omega l F_{211}+\ldots\right] .
\end{aligned}
$$

The groove profile governs the intensity distribution among the different orders $k$. Most common are laminar gratings, where the cross section thru the surface is of rectangular shape. The ratio of groove width to period and the groove height are used to suppress the second order radiation and maximize the first order efficiency at a particular wavelength, respectively. Similarly a tooth shape cross section (blaze) enhances the first order efficiency even further.

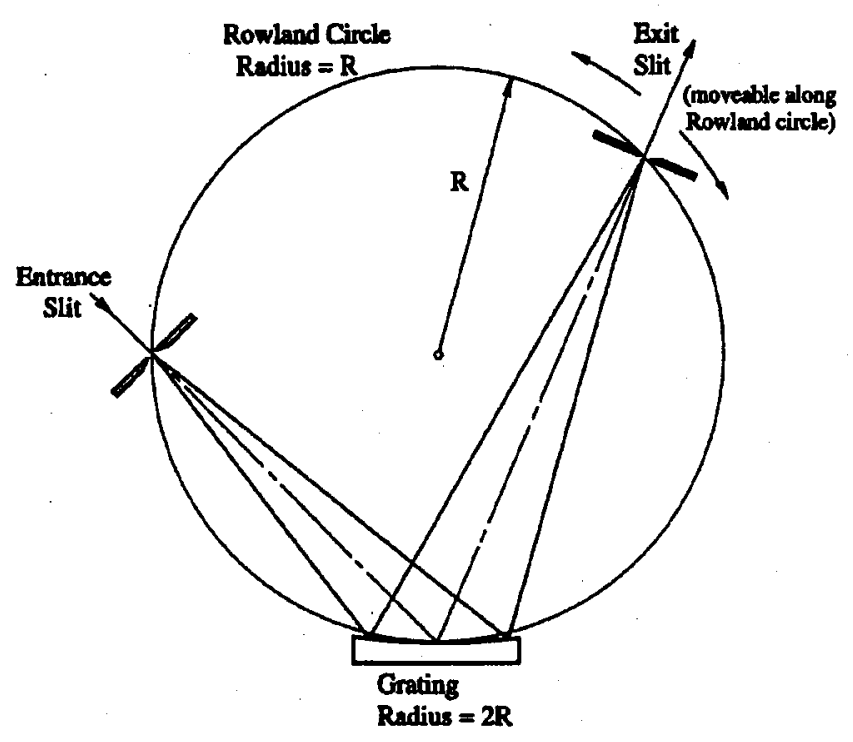

Fig. 3. The Rowland circle for a spherical grating.

Tuning the wavelength requires a mechanical motion of the grating which in general will change the focussing. Defocussing is avoided if $F_{200}=0$ in spite of the tuning. For a spherical grating this leads to the Rowland circle [6] (see Fig. 3).

\section{Monochromators}

Simple monochromators employing only the grating in the dispersive section between fixed entrance and exit slit can be in focus only at one or two wavelengths. Introducing a second moving optical element into the dispersive part originally proposed by Petersen et al. [7] allows us to keep the focus at the (fixed) exit slit (see Fig. 4). The idea has been further developed to do without any elliptical mirror [7]. This idea is also applied for the focusing spherical grating monochromator (FSGM) [8]. A high resolution exceeding 10000 is achieved using plane and spherical optical elements only. 


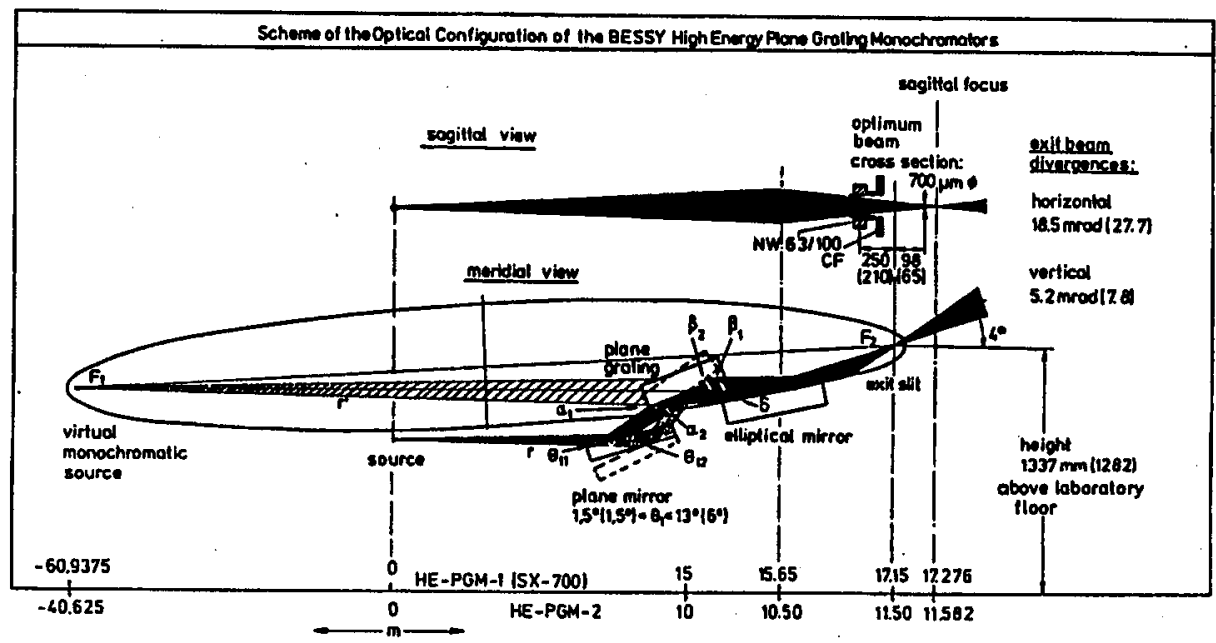

Fig. 4. The optical layout of the Petersen plane grating monochromator SX-700 (numbers in parenthesis are for HE-PGM2).

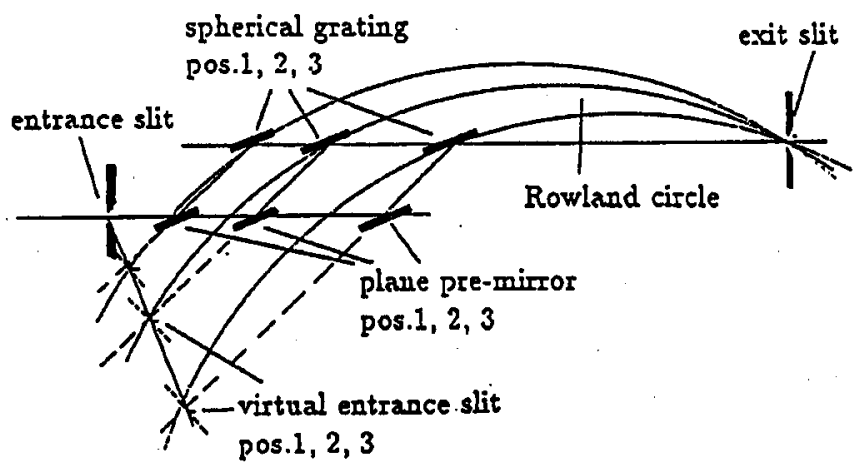

Fig. 5. Optical configuration of the LBL/BESSY constant length Rowland circle monochromator.

The constant length monochromator [9] is an alternative solution to the Rowland circle (see Fig. 5): while the grating rotates around an axis in the grating surface it translates between entrance and exit slit together with a plane mirror.

An interesting idea is to allow for a variation of the grating line density along the length of the grating $[10,11]$. This leads to a compact (smaller than $2 \mathrm{~m}$ long), easy to use (only one rotation of the grating), high resolution (third highest resolution of the $\mathrm{N}_{2}$ vibrational spectrum) beam line.

\section{Further topics}

In this section we collect some topics which the authors believe to be of importance in VUV beam line design and usage. 


\subsection{Heatload}

A large part of the optical power is absorbed in the mirror surface and converted into heat. Depending on the ratio of the thermal expansion coefficient and the thermal conductivity of the substrate material a deformation occurs. Different strategies have been introduced to overcome the problem. SiC is a suitable substrate material with the best figure of merit. The largest heat load can be absorbed in a mirror that is of minor importance for maintaining the (vertical) brilliance. The substrate is cooled from the bottom or from the side. Such cooling schemes can be designed to be uniform or matched to the heat distribution [12]; the latter one awaits an experimental verification.

\subsection{Ray tracking}

Ray tracking codes based on geometric optics are available [13]. Such codes track a large number of rays thru the optical system and provide information on the intensity distribution in selected planes. Diffraction occurring at the grating are included while diffraction occurring at apertures are usually not. The latter ones are expected to become increasingly important for beam line design and usage because of the limited source in the vertical plane diffraction. In recent times several attempts have been undertaken to trace amplitude and phase thru the system. Experiences at BESSY [14] show first promising results.

\subsection{Multilayers}

The extension of dielectric coating, well-known in the visible part of the spectrum, to the VUV is faced with a number of technical and physical problems: absorption limits the useful number of layers. An interfacial roughness of a few atomic layers representing the state of the art of thin film technology becomes less and less acceptable the shorter the wavelength. Material combinations must be found that form smooth interfaces and show little or no interdiffusion. Multilayers are chromatic elements. A typical multilayer in the VUV consists of hundred layers with a spacing of around $3 \mathrm{~nm}$. Among the material combinations that have been used are $\mathrm{Mo} / \mathrm{Si}, \mathrm{Cr} / \mathrm{C}$, and $\mathrm{W} / \mathrm{C}$. Mo/Si forms almost perfect multilayers that can be used up to the Si $L$-edge at $99 \mathrm{eV}$.

A so-called Bragg-Fresnel lens consists of a multilayer operating close to normal incidence which has circular diffracting structures like an ordinary zone plate. It acts as a monochromatising highly reflecting focussing mirror. An application of such a system made from a 130 period $\mathrm{W} / \mathrm{C}$ multilayer to monitor the electron beam cross section at $189 \mathrm{eV}$ has been reported [15].

\subsection{Small spot}

A wide field going well beyond this contribution is related to high spatial resolution. The scientific reward is expected to justify the experimental efforts. A major progress comes from the availability of high brilliant SR sources. 


\section{Outlook}

It is not expected that the growth rate of the application of SR to scientific and technical problems will stay at the same high level as in the past. However, many important problems will require SR. It is the task of the beam line designer to provide optimized sources of radiation, and it is the task of the scientific user to make optimised use of the expensive tool.

\section{References}

[1] W.B. Peatman, Mirrors, Grating, and Slits, Gordon and Breach, 1997, to be published.

[2] J.A.R. Samson, Techniques of Vacuum Ultraviolet Spectroscopy, Wiley, New York 1967.

[3] M. Born, E. Wolf, Principle of Optics, Pergamon Press, Oxford 1980.

[4] H. Noda, T. Namioka, M. Seya, Jpn. J. Appl. Phys. 15, 1181 (1976).

[5] H.G. Beutler, J. Opt. Soc. Am. 35, 311 (1945); see also: H. Haber, J. Opt. Soc. Am. 40, 153 (1950); H. Hogrefe, M.R. Howells, E. Hoyer, SPIE Proc. 733, 274 (1986).

[6] H.A. Rowland, Philos. Mag. 13, 469 (1982); ibid. 16, 197 (1983).

[7] H. Petersen, Ch. Jung, Ch. Hellwig, W.B. Peatman, W. Gudat, Rev. Sci. Instr. 66, 1 (1995).

[8] W.B. Peatman, J. Bahrdt, F. Eggenstein, G. Reichardt, F. Senf, Rev. Sci. Instr. 66, 2801 (1995).

[9] F. Senf, F. Eggenstein, W.B. Peatman, Rev. Sci. Instr. 63, 1326 (1992).

[10] M.C. Hettrick, J.H. Underwood, P.J. Batson, M.J. Eckart, Appl. Opt. 27, 200 (1988).

[11] T. Harada, M. Itou, T. Kita, SPIE Proc. 503, 114 (1984).

[12] U. Menthel, W.B. Peatman, F. Senf, Rev. Sci. Instr. 63, 481 (1992).

[13] J. Feldhaus, F. Schäfers, Beschreibung des Raytracing-Programmsystems RAY, BESSY, Berlin, private communication, for further information contact F. Schäfers at BESSY.

[14] J. Bahrdt, private communication and to be published.

[15] K. Holldack, A. Erko, T. Noll, W.B. Peatman, BESSY Annual Report 1994, 440 (1994). 